\title{
Active tactile sensing in a neurorobotic Braille-reading system
}

\author{
Luca Leonardo Bologna*, Jérémie Pinoteau*, Jesús Garrido and Angelo Arleo
}

\begin{abstract}
We present a neuro-inspired system for the investigation of fine dynamic haptic discrimination for neurorobotic and neuroprosthetic applications. A Braille reading task is adopted as case study. First, tactile inputs are encoded at the level of primary afferents mimicking human mechanoreceptors. Then, a network of simulated second-order neurones processes these primary signals prior to their transmission to a downstream classifier. The latter estimates the likelihood distribution of all Braille characters which is used to determine the letter being read. We also investigate how this distribution could be used to regulate the fingertip acceleration to maximise Braillereading performances. We employ the spiking neural network paradigm to model first- and second-order neural responses, and apply an information theoretical analysis to measure the neurotransmission reliability of the spiking patterns from peripheral to more "central" areas of the system.
\end{abstract}

Our results show that the firing patterns of first- and secondorder responses convey enough information to achieve an offline perfect discrimination of the entire Braille alphabet as rapidly as $250 \mathrm{~ms}$ after the occurrence of the first spike. Furthermore, $89 \%$ of the scanned characters are correctly recognised during an online Braille reading task at constant velocity. Finally, we show that the class probability distributions obtained during reading, can be used to optimise the scanning velocity.

Index Terms - Tactile information coding, Dynamic haptic discrimination, Braille reading, Primary tactile afferents, Second order cuneate neurones, Artificial touch sensor, Bayesian classifiers, Metrical information theory.

\section{INTRODUCTION}

During haptic exploration, information about the environment is conveyed through the forces that are applied to the skin of the hand, notably to the fingertips which constitute the most sensitive of its parts and are prominently involved in object manipulation/recognition tasks. Mechanoreceptors innervating the epidermis are stimulated by the mechanical indentations and deformations of the skin, and transmit the information to the spinal cord and to the cuneate nucleus $(\mathrm{CN})$ of the brainstem. The $\mathrm{CN}$ projects to several areas of the central nervous system, including the cerebellum and the thalamus, which in turn projects to the primary somatosensory cortex. Processing along this pathway allows haptic information to be interpreted and leads to adaptive motor responses, such as in the case of object manipulation and tactile exploration.

${ }^{*}$ L.L.B. and J.P. contributed equally to this work.

Corresponding author: L.L.B., luca.bologna@upme. fr

Luca Leonardo Bologna, Jérémie Pinoteau and Angelo Arleo are with the Adaptive NeuroComputation Group, Unit of Neurobiology of Adaptive Processes, UMR 7102, CNRS-University Pierre and Marie Curie P6, 75005 Paris, France

Jesús Garrido is with the Department of Cellular-Molecular Physiological and Pharmacological Sciences, University of Pavia, 27100, Pavia, Italy
So far, very few works modelled the activity of primary afferents in monkeys [1], [2] and humans [3] and computational studies on second order cuneate neurone networks are lacking [4]. Also, to the best of our knowledge, no extensive investigation has been carried out on the transmission of neural signals through the multi-stage coding mechanisms from the periphery to central areas of the nervous system.

Here, we propose a neurorobotic framework to study active sensing during a fine touch discrimination task with the aim of shedding light on the principles underlying neurotransmission in humans for improving neurorobotics and neuroprosthetics. We take into account the encoding/decoding process of neural signals occuring at the first and second order somatosensory stages and we test how well a probabilistic decoder can discriminate the stimuli given the second order network output

We simulate skin indentation protocols in which Braillelike tactile characters are dynamically scanned by an artificial touch sensor. Analogue deformation signals act as inputs to a network of leaky-integrate-and-fire neurones (LIF), which perform an analogue-to-spike conversion and mimick the role of cutaneous mechanoreceptors. In particular, we model the activity of Slow Adapting type I (SA-I) mechanoreceptors, in terms of both spiking discharge and receptive fields (see [5], for a recent review). The population of LIF neurones projects onto a network of second order units modelling $\mathrm{CN}$ responses. We employ the Spike Response Model (SRM) [6] to capture the stochastic nature of unitary cuneate responses. Downstream from the CN network, a naive Bayesian classifier computes the probability distribution of all Braille characters online, on the basis of the ongoing cuneate population activity. The likelihood distributions provided by the Bayesian classifier are ultimately used to discriminate the letter currently scanned and to devise an adapted velocity trajectory optimising the scanning/discrimination time.

\section{Material And Methods}

Figure 1 shows the complete robotic setup used for the Braille scanning task. We use a set of 26 different probes reproducing a scaled version (1:1.67) of all Braille characters (Fig. 1B) to stimulate an artificial touch sensor (simulating the human fingertip deformations exerted by Braille dots). The touch sensor is rubbed over all Braille characters at constant velocity. Its analogue responses are encoded and decoded by the simulated first- and second-order afferents (mechanoreceptors and cuneate neurones respectively), prior to the "high-level" probabilistic classification. 
A

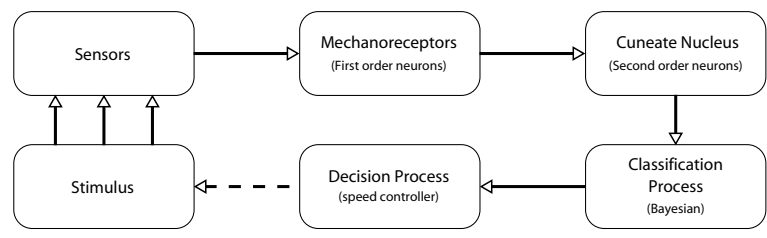

B

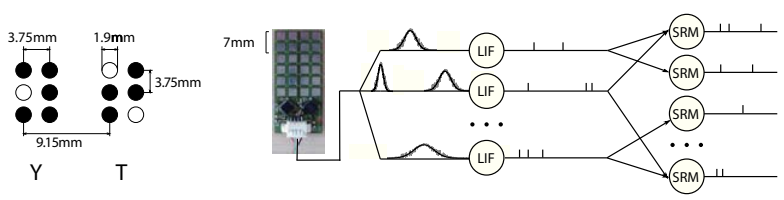

C

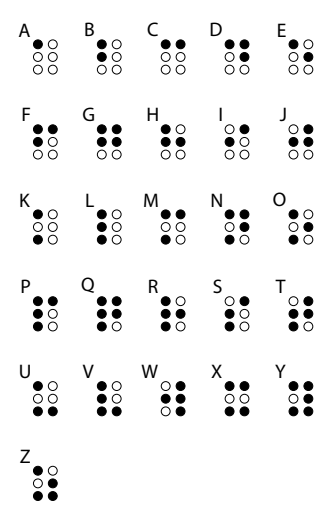

D

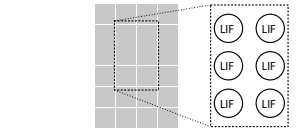

Sensor-to-mechanoreceptor mapping
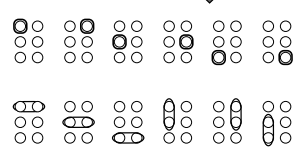

$\begin{array}{llllll}\circ & 28 & 00 & 88 & 00 \\ \circ & 00 & 88 & 00 & 28\end{array}$

CN neuron receptive fieldfs

$E$
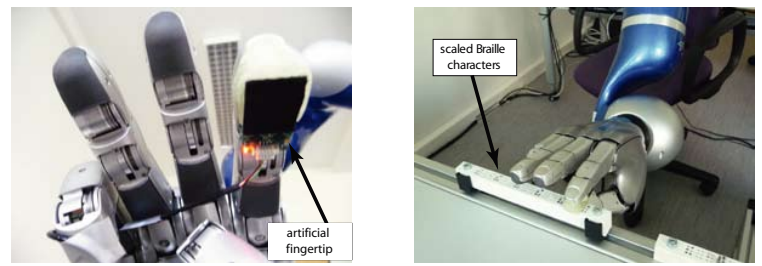

Fig. 1. Overview of the entire encoding/decoding pathway and robotic setup. (A) Following the loop, starting at the top left: we employ Braille characters as tactile stimuli to indent a capacitive artificial touch sensor. The analogue responses provided by the touch sensor drive a network of LeakyIntegrate-and-Fire (LIF) neurones [7] converting analogue signals into spiking activity and mimicking fingertip mechanoreceptors. The population of LIF neurones projects onto a network of Spike-Response-Model units [6] implementing second order cuneate nucleus $(\mathrm{CN})$ cells of the brainstem. The outgoing activity is decoded using a Naive Bayesian Classifier whose output allows a speed controller to devise an improved velocity for the fingertip's movement. (B) From left to right: Scaled Braille characters used as stimuli; artificial fingertip (without its neoprene layer); LIF and SRM neurones with their outgoing spiking activity. (C) Braille alphabet. (D) LIF neurones modelling mechanoreceptor activity fulfil a topological mapping of fingertip regions (top). $\mathrm{CN}$ cell receptive fields are built so as to collect activity of either a single cell or different possible combinations of two or three adjacent mechanoreceptors (bottom). (E) The artificial fingertip mounted on a robotic hand/arm setup (c) Institute of Robotics and Mechatronics, German Aerospace Center).

\section{A. The artificial touch sensor}

An artificial skin prototype ${ }^{1}$ [8], [9] was initially used to collect and characterise a first dataset of analogue responses to Braille character indentations. This artificial fingertip consists of 24 capacitive square sensors disposed according

\footnotetext{
${ }^{1}$ Developed at the Italian Institute of Technology (IIT), Genoa, Italy.
}

to a rectangular grid layout. Each sensor has a dimension of approximately $3 \mathrm{~mm}$ and the inter-centre distance is $4 \mathrm{~mm}$ (Fig. 1B), for a total sensitive surface of approximately $18 \times$ $23 \mathrm{~mm}$. The array is covered by a $2.5 \mathrm{~mm}$ thick neoprene layer in order to modulate the pressure exerted over the sensors. The response strength of each sensor, proportional to the indentation level, ranges between 0 and 189 femtoFarads (fF). The acquisition frequency of the capacitive sensor is $20 \mathrm{~Hz}$. During the Braille reading experiments, the touch sensor outputs varied between 0 and $60 \mathrm{fF}$, and its receptive fields extended up to a $1.6 \mathrm{~mm}$ radius (depending on the stimulation pattern).

We developed a simulator reproducing the responses of the artificial fingertip and offering a greater flexibility in data generation and experimental protocols [9]. We modelled the touch sensor responses by means of Gaussian kernels of amplitude $55 \mathrm{fF}$ and standard deviation $1.6 \mathrm{~mm}$. Additionally, we added a white noise to the amplitude and standard deviation of the response $(2.5 \mathrm{fF}$ and $0.1 \mathrm{~mm}$ respectively) and we modelled possible position errors due to the experimental setup by adding a Gaussian noise to the position of each stimulus $(\mathrm{sd}=0.1 \mathrm{~mm})$.

\section{B. Primary afferent coding: analogue-to-spike transduction}

We implement a network of LIF neurones [10], [7] to convert analogue touch sensor outputs into spike train patterns (Fig. 1D). We map the capacitance values provided by the touch sensors into current intensities $I(t)$ driving the LIF neurones by applying a multiplicative gain factor of $-390 \mathrm{pA} / \mathrm{fF}$ (determined by comparing output LIF spike trains against recorded mechanoreceptor responses [11]). The dynamics of the membrane potential $V(t)$ of each LIF neurone is:

$$
C \cdot \frac{d V(t)}{d t}=-g \cdot\left(V(t)-V_{\text {leak }}\right)-I(t)
$$

where $C=0.5 \mathrm{nF}$ denotes the membrane capacitance, $g=25$ $\mathrm{nS}$ the passive conductance, $V_{\text {leak }}=-70 \mathrm{mV}$ the resting membrane potential, and $I(t)$ the total synaptic input of a neurone. The membrane time constant is then $\tau=C / g=20$ ms. Whenever the membrane potential $V(t)$ reaches the threshold $V_{t h r}=-50 \mathrm{mV}$ the LIF neurone emits an action potential. Then, its membrane potential is reset to $V_{\text {reset }}=$ $-100 \mathrm{mV}$ and the dynamics of $V(t)$ are frozen during a refractory period $\Delta t_{\text {ref }}=2 \mathrm{~ms}$. We also use a "threshold fatigue" [7] to model the phenomenon of "habituation". It consists in increasing the threshold $V_{t h r}$ by a value $A_{t h r}$ each time the neurone discharges, making it harder for the neurone to spike again (i.e. preventing it from responding in a highly tonic manner even in the presence of strong inputs). In the absence of spikes, the threshold decreases exponentially back to its resting value $V_{\text {rest Thr }}$ :

$$
\frac{d V_{t h r}(t)}{d t}=-\frac{V_{t h r}(t)-V_{r e s t} T h r}{\tau_{t h r}}
$$

with $\tau_{t h r}=100 \mathrm{~ms}, V_{\text {rest Thr }}=-50 \mathrm{mV}$ and $A_{t h r}=50 \mathrm{mV}$. 


\section{Second-order processing in the cuneate network}

We model single cuneate responses by means of the previously presented SRM model [6], [12] able to capture the stochastic nature of the $\mathrm{CN}$ cells (unpublished data by $\mathrm{H}$. Jörntell). For the implementation, we adopted a previously presented computational framework [13]. We include a noise model (i.e. escape noise) that follows a stochastic process, so providing a linear probabilistic neuronal model.

An input spike arrival at time $t$ induces a membrane potential depolarisation $\Delta V(t)$ described by:

$$
\Delta V(t) \propto \sqrt{t} \exp (-t / \tau)
$$

where the parameter $\tau=2 \mathrm{~ms}$ determines the decay time constant of the EPSP (excitatory postsynaptic potential). If several afferent spikes excite the neurone within a short time window, then the EPSPs sum up linearly:

$$
V(t)=V_{r}+\sum_{i, j} w_{i} \Delta V\left(t-\hat{t}_{i}^{j}\right)
$$

where $i$ denotes presynaptic neurones, $j$ indexes the spikes emitted by a presynaptic neurone $i$ at times $\hat{t}_{i}^{j}$, and $V_{r}=$ $-70 \mathrm{mV}$ is the resting potential. The term $w_{i}$ indicates the synaptic weight of the projection from the presynaptic unit $i$, defined as:

$$
w_{i}=W \cdot w_{i}^{0,1}
$$

with factor $W$ determining the upper bound of the synaptic efficacy, and $w_{i}^{0,1}$ being constrained within the range $[0,1]$. We use $W=0.04$ in our simulations. At each time step, a function $g(t)$ computes the instantaneous firing rate of the cell according to:

$$
g(t)=r_{0} \log \left(1+\exp \left(\frac{V(t)-V_{0}}{V_{f}}\right)\right)
$$

where the constants $r_{0}=11 \mathrm{~Hz}, V_{0}=-65 \mathrm{mV}, V_{f}=0.1 \mathrm{mV}$ are the instantaneous firing rate, the probabilistic threshold potential, and a gain factor, respectively. A function $A(t)$ determines the refractoriness property of the neurone:

$$
A(t)=\frac{\left(t-\hat{t}-\tau_{a b s}\right)^{2}}{\tau_{r e l}^{2}+\left(t-\hat{t}-\tau_{a b s}\right)^{2}} \mathscr{H}\left(t-\hat{t}-\tau_{a b s}\right)
$$

where $\tau_{a b s}=3 \mathrm{~ms}$ and $\tau_{\text {rel }}=9 \mathrm{~ms}$ denote the absolute and relative refractory periods, respectively, $\hat{t}$ the time of the last spike emitted, and $\mathscr{H}$ the Heaviside function. Finally, the functions $g(t)$ and $A(t)$ allow the probability of firing $p(t)$ to be computed:

$$
p(t)=1-\exp (-g(t) A(t))
$$

We implement the synaptic connections between mechanoreceptors and $\mathrm{CN}$ neurones so as to generate the receptive fields shown in Fig. 1D. Each $\mathrm{CN}$ neurone receives non-plastic inputs from either one or a group of two/three adjacent mechanoreceptors depending on the stimulus (see the caption of Fig. 1 for details). The dimension and shape of the receptive fields and the synaptic weight distribution of the mechanoreceptor-to- $\mathrm{CN}$ projections allow topographical information to be maintained at the level of the second order output space. Also, thanks to the adopted connectivity layout, CN neurones collecting signals from large receptive fields mirror both single primary neurone activation and multiple co-activations, thereby enriching the population spiking dynamics.

\section{Assessing neurotransmission reliability: metrical infor-} mation analysis

To decode neural activities and quantify fine touch discrimination, we applied the recently defined metrical mutual information $I^{*}(R ; S)$ [14]. Unlike Shannon's traditional definition of mutual information [15], [16], this measure takes into account the metrical properties of the spike train space [17], [18], [19] and it has been proven to be suitable to decode the responses of human mechanoreceptors obtained via microneurography recordings [12], [14]. The definition of $I^{*}(R ; S)$ relies on a similarity function based on the distance between spike train responses elicited by the same stimulus and by different stimuli. The Victor and Purpura Distance was used in the definition of the metrical information [17]. This specific spike train metrics makes it possible to modulate -through a cost parameter- the importance given to temporal (and rate) coding in the mapping of the spike train space.

The perfect discrimination condition corresponds to maximum $I^{*}(R ; S)$ and zero conditional entropy $H^{*}(R \mid S)$ [14]. It occurs when the size of the largest cluster of responses becomes smaller than the smallest distance between all clusters of responses [14].

\section{E. Online classification of Braille characters: Naive Bayesian Classifier}

In order to discriminate Braille characters during the reading task, we trained a Naive Bayesian Classifier (NBC) via multinomial distributions. This learning algorithm belongs to the family of probabilistic classifiers relying on Bayes' rule to compute the posterior probability of the sample classes. Despite its simplicity, the NBC has been proven to be fast and efficient even when the feature independence hypothesis underlying its application is not fulfilled [20], [21].

Braille characters (i.e. dataset classes) are defined from the spike train activity encoded after the touch sensor signals. With the aim of performing a fast classification, we built the training dataset by binning the activity of the $49 \mathrm{CN}$ neurones with temporal windows of increasing lengths (see Fig. 3A for an example). The bin size increment was fixed at 10 ms. Each temporal bin is characterised by the spatiotemporal organisation of the firing activity it encloses and it can be labelled with the corresponding character. The arrays of 49 spike counts for each temporal bin (features) and their corresponding characters (classes) form the complete dataset.

\section{F. From classification to reading velocity control: Kurtosis- based assessment of the likelihood distribution}

Given the procedure adopted to build the activity dataset and the small time bin chosen as temporal increment, characters' probability distributions can be measured frequently 
while reading. We use such information to compute the excess Kurtosis index which indicates to what extent a probability distribution is peaked around its values. We investigate whether the variation of the Kurtosis can be used to modulate the finger scanning velocity in order to effectively decrease classification time with respect to a constant speed movement.

\section{RESULTS}

\section{A. Characterisation of mechanoreceptor responses}

A previous study has compared some characteristics of the simulated and human mechanoreceptor responses to fingertip skin indentation caused by Braille-character probes [3]. The simulated primary afferent responses exhibit receptive fields qualitatively similar to those of real Slow Adaptive I (SA-I) mechanoreceptors, in terms of shape, dimensions and signalto-noise ratio (see Fig. 2A). SA-I units, along with -to a slightly lesser extent- Fast Adaptive type I (FA-I) primary afferents, show a topological mapping (i.e. their activity correlates with the area of stimulation), demonstrating their role in encoding spatial discontinuities [5]. There is no clear experimental evidence on whether SA-I or FA-I mechanoreceptors primarily carry the information needed for Braille character recognition [22].

A comparison between the first spike jitter distributions of the model primary responses and that of their biological counterparts (SA-I units) show them to be statistically equivalent (Mann-Whitney $\mathrm{U}$ test $P>0.11$; KolmogorovSmirnov test $P>0.076$ ) in terms of both median and shape, despite a time lag in the simulated responses of about 2 $\mathrm{ms}$ (see Fig. 2B), given to the adopted sensor simulation frequency. Thus, modelled mechanoreceptors present the same variability in spike latencies as SA-I afferents, but on a larger time scale. The comparison of inter-spike intervals (ISI) distributions, however, reveals that the spike trains of model neurones lack the ISI variability of those recorded in humans, although a Mann-Whitney $U$ test shows that both distribution medians are equivalent, $P>0.16$ (data not shown). The difference in ISI variability may be due to the viscoelastic properties and more complex dynamics of the human skin compared to the artificial finger.

\section{B. Information content of afferent responses}

We perform an information theoretical analysis to decode simulated first and second order responses to 26 distinct Braille characters sensed during a scanning task. We focus on the evolution of information over time and quantify how rapidly perfect discrimination of all Braille stimuli can be achieved after stimulus onset.

Fig. 2C top illustrates the evolution of the metrical information and conditional entropy upon indentation by Braille stimuli while scanning at $30 \mathrm{~mm} / \mathrm{s}$. The cost in the Victor and Purpura Distance was chosen as the parameter value allowing the earliest possible perfect discrimination. At the first order afferent level, first spikes occur at around $100 \mathrm{~ms}$, and $250 \mathrm{~ms}$ later the condition for an errorless stimulus reconstruction is satisfied. In comparison, a small delay is observed at the $\mathrm{CN}$

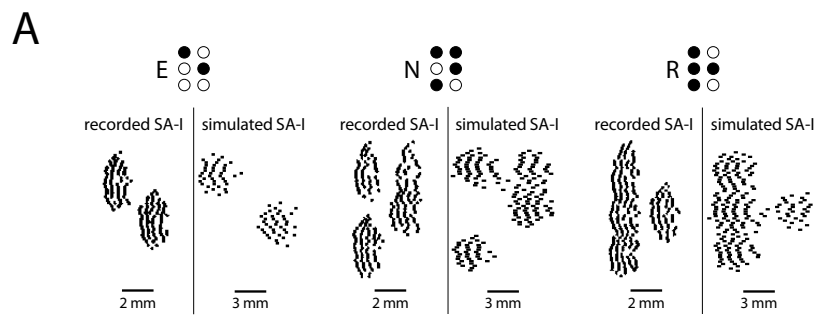

B
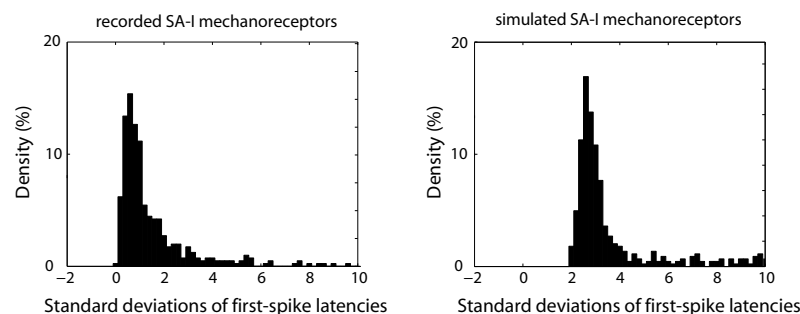

C

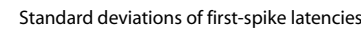

Standard deviations of first-spike latencies
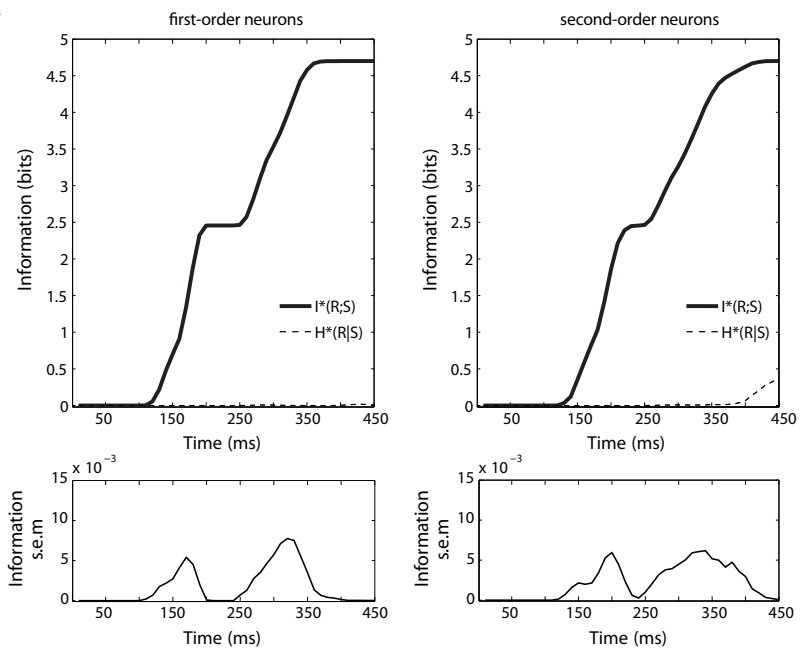

Fig. 2. Characterisation of mechanoreceptor responses and theoretical information analysis of primary and secondary afferent responses to Braille stimuli. (A) Spatial event plots of human SA-I mechanoreceptor responses to scanned Braille characters 'e', 'n' and 'r' compared to that of their simulated counterparts (recorded sections adapted from [22]). (B) Distribution of standard deviations (SD) of first-spike latencies for both SA-I (left) and simulated (right) mechanoreceptor responses to comparable stimuli. (C) Top: Time course of metrical information (full line) and conditional entropy (dashed line) at the output of the LIF neurones (left) and of the CN model (right), as the fingertip travels over the Braille characters at $30 \mathrm{~mm} / \mathrm{s}$. The 26 Braille characters serve as stimuli, with 20 repetitions per stimulus used. First spikes occure at around $100 \mathrm{~ms}$, and the perfect discrimination condition is reached about $250 \mathrm{~ms}$ later. Bottom: information variability, measured as mean standard error, over time.

output level, and almost perfect discrimination is possible just as soon. As expected, the metrical information curve exhibits a plateau starting at around $200 \mathrm{~ms}$ and lasting a little over $75 \mathrm{~ms}$. This corresponds to the stimulation phase during which the first column of Braille dots enters in contact with the fingertip while the second does not stimulate any sensor yet. The information value at plateau is about half of the total amount of information transmitted (Fig. 2C). 
A

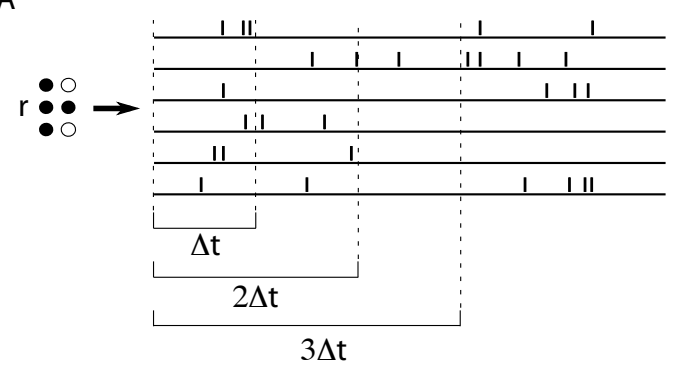

B

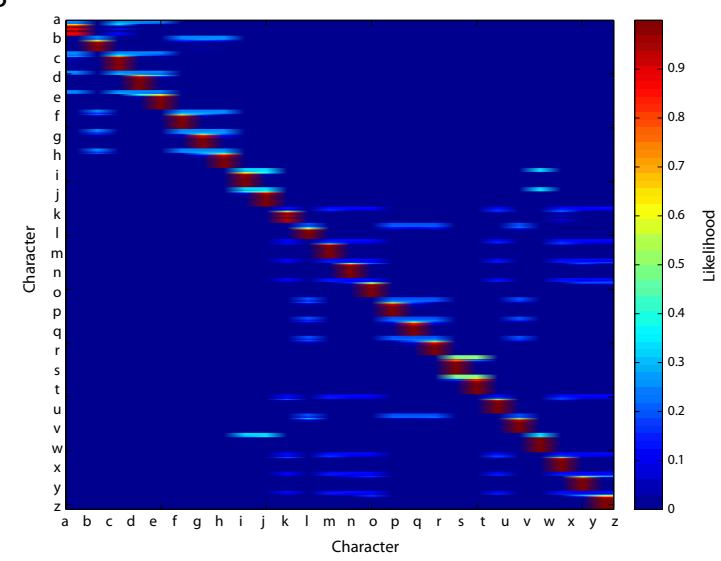

C
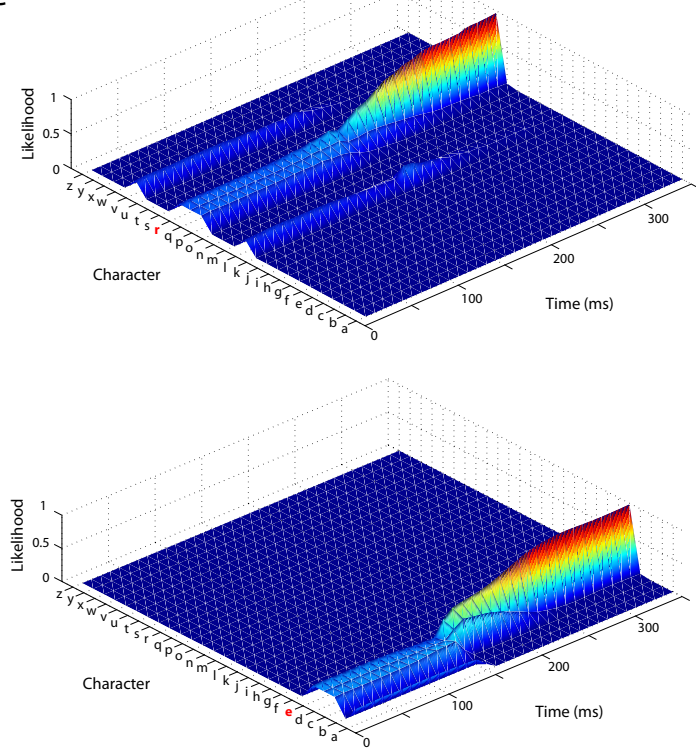

Fig. 3. Naive Bayesian classifier output likelihood distributions. (A) CN spiking activity is binned following time windows of increasing size $(n \times \Delta t)$. (B) Probabilities for responses to a Braille stimulus (rows of matrix) of being classified as those of a given stimulus (columns of matrix), as determined by the probabilistic classifier. The responses to each character is binned following multiple time windows of increasing size (as described in panel A). Small bins capture little activity and are more likely than larger bins to be wrongly recognized. (C) Expanded sections of panel B for letters ' $r$ ' and 'e'. Initially, different characters seem to be equally probable. As time increases, bin size increases and the probability distribution converges around one character, which characterises the unique likely stimulus.

\section{Classification of Braille characters in a reading task}

We collect a dataset of character samples and fit a probabilistic classifier to estimate the occurrence probability of the entire set of Braille characters while reading. When the NBC fitting parameters are used for offline classification, a near perfect discrimination is possible. In fact, as one can observe in Fig. 3B, diagonal values of the classification confusion matrix are close to one. At the same time, given to the binning procedure adopted, data samples built by gathering the spiking activity in small temporal windows (cf. II-E) do not carry enough information to allow a correct discrimination. A clear example of the probability evolution distributions over time is given in Fig. 3C. At the beginning of the scanning movement, the spiking activity of the $\mathrm{CN}$ neurones responding to letter ' $\mathrm{r}$ ' ( $3 \mathrm{C}$, top) does not allow to distinguish between ' $r$ ' and the other Braille characters with a similar spatial dot configuration (i.e., 'l', 'p', 'q', 'v'. See Fig. 1C). But, as time evolves, the uncertainty decreases till a correct classification is possible (maximal probability reached for letter ' $r$ '). A similar example can be observed in Fig. 3C, bottom (letter 'e' scanning). We tested the system with online simulations by brushing the fingertip over the characters at the constant scanning velocity of 30 $\mathrm{mm} / \mathrm{s}$. In this scenario, the classifier correctly discriminated $89 \%$ of the scanned characters $(10 \%$ false positives, $1 \%$ no classification).

\section{Online reading velocity modulation}

The methodology we adopted for building the spikingactivity based dataset, lends itself to a frequent computation of Braille character probability distributions. We asked whether the probabilities evolution over time could be seen as a possible mechanism underlying the changes in reading speed observed in blind subjects [23].

We observed that, when the Kurtosis index gradient (normalized by a constant factor) is applied to modulate the reading velocity at each time step, the result is an overall improvement in the reading performance. In fact, at the end of a single character scanning, the average velocity adopted with an active speed modulation, is higher than the velocity the finger had when it first encountered the character (and that would have been maintained if no changes had been applied). An example of kurtosis gradient and dynamically modulated velocity is given in Fig. 4.

\section{Discussion}

Dynamic haptic discrimination in humans involves several processes at different levels of abstraction (e.g. encoding/decoding of afferent signals, sensorimotor control, decision making).

In this study, we investigate active sensing by integrating tactile information coding at the neuronal level with a probabilistic framework for dynamic tactile stimuli discrimination. In a Braille reading task implementation, we convert the analogue signals from an artificial fingertip into spiking activity and we apply an information theoretical analysis to the first and second stage afferent output. We finally interpret online the output of the second order Cuneate Nucleus neurones through a Naive Bayesian Classifier and 
A

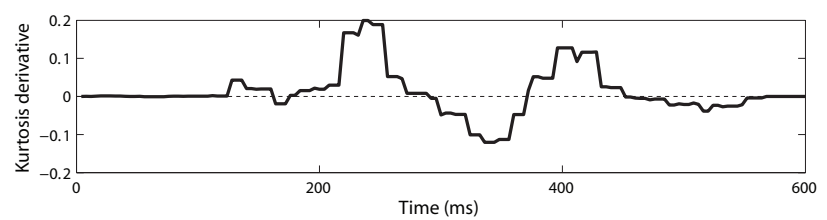

B

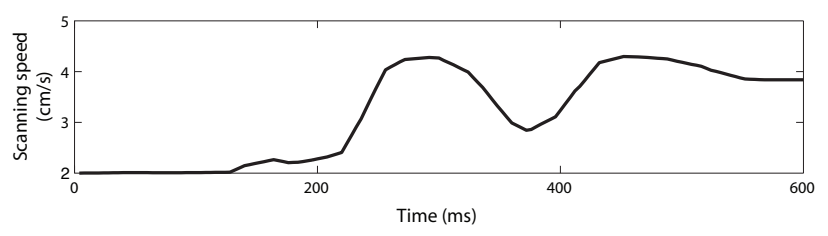

Fig. 4. Kurtosis of probability distribution and resulting speed modulation. (A) Evolution of the kurtosis of the posterior probabilities distributions computed by the naive Bayesian classifier while scanning the letter B. (B) Time course of the velocity modulation as a function of time.

ask whether the character probability distribution can be used for determining an efficient reading velocity.

We show that signals at the earliest stages of the haptic ascending pathway are conveyed as to allow a complete and fast discrimination of inputs by downstream decoders. We demonstrate that a probabilistic approach allows to efficiently discriminate all Braille characters in an online Braille reading task and we finally argue that the level of uncertainty of the reader about the character being read, could determine an optimal reading velocity.

We are currently investigating how the interferences created by different scanning velocities on the activity of $\mathrm{CN}$ neurones influence the classification process. We are also testing the speed modulation paradigm by closing the loop and allowing an online control of the fingertip scanning velocity. So doing, we will be able to observe whether an increase in the mean reading velocity is present and how the classification performances are affected by a dynamical variation in velocity. We finally propose to investigate if the speed modulation can help improve discrimination performance in the case of noisy fingertip movements.

\section{ACKNOWLEDGMENTS}

Granted by the EC Project SENSOPAC (no. IST-028056IP) and by the French Direction Générale de l'Armement (DGA). The authors also thank the CASPUR Consortium (www.caspur.it) for providing the high performance computing facilities.

\section{REFERENCES}

[1] A. W. Freeman and K. O. Johnson, "Cutaneous mechanoreceptors in macaque monkey: temporal discharge patterns evoked by vibration, and a receptor model", The Journal of physiology, vol. 323, no. 1, pp. 2141, 1982.

[2] S. S. Kim, A. P. Sripati and S. J. Bensmaia, "Predicting the timing of spikes evoked by tactile stimulation of the hand", Journal of Neurophysiology, vol. 104, no. 3, pp. 1484-1496, Sept. 2010.

[3] L. L. Bologna, J. Pinoteau, R. Brasselet, M. Maggiali, and A. Arleo, "Encoding/decoding of first and second order tactile afferents in a neurorobotic application", Journal of Physiology-Paris, vol. 105, no. 1-3, pp. 25-35, June 2011.
[4] E. Sánchez, S. Barro, J. Mariño and A. Canedo, "Cortical modulation of dorsal column nuclei: A computational study", Journal of Computational Neuroscience, vol. 21, no. 1, pp. 21-33, Apr. 2006.

[5] R. S. Johansson and J. R. Flanagan, "Coding and use of tactile signals from the fingertips in object manipulation tasks", Nature Reviews Neuroscience, vol. 10, no. 5, pp. 345-359, May 2009.

[6] W. Gerstner and W. Kistler, Spiking Neuron Models, Cambridge University Press, 2002.

[7] M. J. Chacron, K. Pakdaman and A. Longtin, "Interspike interval correlations, memory, adaptation, and refractoriness in a leaky integrateand-fire model with threshold fatigue", Neural Computation, vol. 15 , no. 2, pp. 253-278, Feb. 2003.

[8] G. Cannata, M. Maggiali, G. Metta and G. Sandini, "An embedded artificial skin for humanoid robots", in Proc. IEEE International Conference on Multisensor Fusion and Integration for Intelligent Systems MFI 2008, 2008, pp. 434-438.

[9] L. L. Bologna, R. Brasselet, M. Maggiali and A. Arleo, "Neuromimetic encoding/decoding of spatiotemporal spiking signals from an artificial touch sensor", in Proc. The 2010 International Joint Conference on Neural Networks (IJCNN), Barcelona, Spain, July 2010 pp. 1-6.

[10] L. Lapicque, "Recherches quantitatives sur l'excitation électrique des nerfs traitée comme une polarisation", Journal de Physiologie et Pathologie General, vol. 9, pp. 620-635, 1907.

[11] R. S. Johansson and I. Birznieks, "First spikes in ensembles of human tactile afferents code complex spatial fingertip events", Nature Neuroscience, vol. 7, no. 2, pp. 170177, Feb. 2004

[12] R. Brasselet, R. Johansson, A. Arleo, Y. Bengio, D. Schuurmans, J. Lafferty, C. K. I. Williams and A. Culotta, "Optimal context separation of spiking haptic signals by second-order somatosensory neurons", in Advances in Neural Information Processing Systems 22, pp. 180-188. 2009.

[13] R. R. Carrillo, E. Ros, C. Boucheny and O. J-M. D Coenen, "A realtime spiking cerebellum model for learning robot control", BioSystems, vol. 94, no. 1-2, pp. 18-27, Nov. 2008.

[14] R. Brasselet, R. S. Johansson and A. Arleo, "Quantifying neurotransmission reliability through metrics-based information analysis", Neural Computation, vol. 23, no. 4, pp. 852-881, Apr. 2011.

[15] E. Shannon, "A mathematical theory of communication", The Bell System Technical Journal, vol. 27, pp. 379-423, 623-656, 1948.

[16] F. Rieke, D. Warland, R. de Ruyter van Stevenick and W. Bialek, Eds. Spikes: Exploring the neural code., Cambridge: MIT Press, 1997.

[17] J. D. Victor and K. P. Purpura, "Nature and precision of temporal coding in visual cortex: a metric-space analysis", Journal of Neurophysiology, vol. 76, no. 2, pp. 1310-1326, Aug. 1996.

[18] S. Schreiber, J. M. Fellous, D. Whitmer, P. Tiesinga and T. J. Sejnowski, "A new correlation-based measure of spike timing reliability", Neurocomputing, vol. 52-54, pp. 925-931, June 2003.

[19] M. C. van Rossum, "A novel spike distance", Neural Computation, vol. 13, no. 4, pp. 751-763, Apr. 2001.

[20] H. Zhang, "The optimality of naive bayes", in FLAIRS Conference. 2004, AAAI Press.

[21] W. Truccolo, G. M. Friehs, J. P. Donoghue and L. R. Hochberg, "Primary motor cortex tuning to intended movement kinematics in humans with tetraplegia", The Journal of Neuroscience, vol. 28, no. 5, pp. $1163-1178$, Jan. 2008.

[22] J. Phillips, R. Johansson and K. Johnson, "Representation of braille characters in human nerve fibres", Experimental Brain Research, vol. 81, no. 3, pp. 589-592, 1990.

[23] B. Hughes, "Movement kinematics of the braille-reading finger", Journal of Visual Impairment \& Blindness, vol. 105, no. 6, pp. 370381, June 2011. 